\title{
Aspects of Dielectric Breakdown in a Model for Disordered Nonlinear Composites
}

\author{
Abhijit Kar Gupta and Asok K. Sen* \\ LTP Section, Saha Institute of Nuclear Physics \\ 1/AF Bidhannagar, Calcutta 700 064, India
}

February 7, 2008

\begin{abstract}
We study dielectric breakdown in a semi-classical bond percolation model for nonlinear composite materials introduced by us and the related breakdown exponent near the percolation threshold in two dimensions. The breakdown exponent after doing finite size scaling analysis is found to be $t_{B} \simeq 1.42$. We discuss in detail the differences in our model from the traditional models for dielectric breakdown and argue that our result seems to be different from the standard result of $4 / 3$ obtained in the previous models.
\end{abstract}

PACS numbers: 64.60.Fr, 64.60.Ht, 77.22.J

${ }^{*}$ Corresponding author, e-mail address: asok@hp2.saha.ernet.in 


\section{Introduction}

Statistical physics of the breakdown of an insulating dielectric into a conducting state (or of a conductor into a 'fused' insulating state) has been the subject of intense research [1] for more than a decade now. Suppose one considers a random binary (two-phase) mixture of metallic and non-metallic components. If the volume fraction of the metallic phase is large enough, the metal phase forms at least one [2] samplespanning cluster in which the non-metallic phase is dispersed in the form of isolated islands. In this regime the electrical conductivity of the sample is large. The system is metallic. On the other hand, for a small volume fraction of the metallic components, the non-metallic phase forms at least one [2] sample-spanning cluster in the presence of small and isolated metallic islands. The system is then in the dielectric or insulating regime. The electrical conductivity of the sample in this regime is ideally zero and extremely small in practice. Now, if one increases the electric field across the sample in this regime, the voltage across the non-metallic bonds keeps increasing and it is not unlikely that some of them may give in to let some current through them or turn metallic. Clearly in this case the breakdown problem is set up with an underlying percolation model.

In the usual dielectric breakdown model [3] of a random mixture of conductors and insulators it is assumed that each insulating bond can withstand a fixed potential difference across it and becomes a conductor if the local potential difference exceeds its threshold. Therefore, the whole lattice is subjected to breakdown at any volume fraction $\left(p<p_{c}\right)$ of conducting components when an appropriately large external voltage, called the breakdown voltage, $V_{B}$, is applied. Its value depends on the specific configuration of the sample, and usually one talks about the configuration-averaged value of $V_{B}$ at any particular $p$. For $p=0$, i.e., when all the bonds are insulators, the breakdown voltage $\left(V_{B}\right)$ scales as the linear size $(L)$ of the lattice: $V_{B} / L=v_{g}$, where $v_{g}$ is the voltage threshold (all insulators are assumed to have an identical 
voltage threshold) for an individual tunnelling bond. For $p \geq p_{c}$, such a lattice is conducting for any small applied voltage and the question of dielectric breakdown does not apply: $V_{B} / L=0$. To remove the trivial system size $(L)$ dependence, we talk about the external breakdown field $\left(E_{B}=V_{B} / L\right)$ instead of the breakdown voltage from now on. The interesting thing happens as one approaches $p \rightarrow p_{c}$ from below. One obtains a criticality and a power law

$$
E_{B} \sim\left(p_{c}-p\right)^{t_{B}}
$$

where $t_{B}$ is called the breakdown exponent. A similar scaling is also known for the mechanical fracture process [4]: $\sigma_{\text {min }} \sim\left(p-p_{c}\right)^{b}$, where $\sigma_{\text {min }}$ is the minimum stress needed to break the system apart.

In general in a breakdown process one defines two critical voltages: one is the breakdown initiation voltage $V_{I}$ at which the nucleation of the breakdown process (akin to an avalanche) is initiated and the other is the final breakdown voltage $V_{F}$ which is the minimum voltage at which the system as a whole breaks apart. In some cases $V_{I}$ is no different from $V_{F}$ [3]. In other cases the system needs some more voltage beyond $V_{I}$ to reach the final breakdown state. It is commented in some earlier works [5, 6] that $V_{I}$ and $V_{F}$ are essentially the same. So the authors of many previous works had actually treated the average value of $V_{I}$ as the average breakdown voltage $\left(V_{B}\right)$. The statistics of $V_{I}$ and $V_{F}$ are also claimed to be the same i.e., they are described by the same critical exponent near the threshold $\left(p_{c}\right)$. There has been a number of works (see e.g. the references [5, 6]) in the literature for estimating the breakdown exponent. A closely related quantity of interest is the mimium-gap path, $g(p)$, of a non-percolating lattice configuration. It is the minimum number of insulating bonds which are to overcome their thresholds to give a connecting path between two opposite sides of a lattice across which the external voltage is applied. The breakdown voltage $\left(V_{B}\right)$ and the minimum gap $(g)$ are actually two different quantities [5] except at $p=0$ and at $p=p_{c}$ although both the quantities near $p_{c}$ seem to behave in the same way and the numerical results [5] claim that their scaling 
exponents are the same near $p_{c}$. It was claimed through an analytical calculation on a hierarchical lattice and through a numerical simulation on a square lattice [7] that the breakdown voltage $\left(V_{B}(p)\right)$ behaves like $g(p)$ in a random lattice. The average of $g(p)$ is supposed to vary as $\left(p_{c}-p\right)^{t_{g}}$, where $t_{g}$ (the minimum-gap exponent) is identified with the breakdown exponent $t_{B}$. Later, it was rigorously established by Chayes et al. [8] in an invasion (or, forced under a pressure) percolation type situation, that $t_{g}=\nu$ in $2 \mathrm{D}$, where $\nu$ is correlation length exponent. This indicates that $t_{B}=t_{g}=\nu$. However, there is a $\log L$ term involved in the scaling relationship of breakdown field $\left(E_{B}\right)$ near percolation threshold $\left(p_{c}\right)$, and $E_{B} \sim \frac{\left(p_{c}-p\right)^{\zeta}}{\ln L}[6]$.

\section{The Model}

We propose a semi-classical (or, semi-quantum) model of percolation [9] which works on the borderline between a classical and a quantum picture. Quantum physics enters our discussion through the possibility of tunnelling of a charge carrier through a barrier (which does not exist classically). Disorder in such systems is known to give rise to 'pinning' or inhibition to transport (say, in charge-density-wave (CDW) systems or flux-vortex lattices of type-II superconductors) upto a critical value of the applied field above which tunnelling is active. Our approach would be to solve an appropriate electrical network based on a semi-classical percolation model. Made of both random resistive and tunnelling elements, this network will be called a random resistor cum tunnelling-bond network (RRTN). There exists a similar model in the literature called a dynamic random resistor network (DRRN) proposed by Gefen et al. [10] to explain the crossover exponent in the experiment on $A u$-films reported in

the same reference. The difference between these two models lies in the fact that the tunnelling elements (or the imperfect insulators) in the DRRN could be anywhere in the non-metallic domain of the system whereas in the RRTN, these elements exist only in the proximity gap between two metallic domains (one can imagine that the charge 
transfer by tunnelling should be most effective only in such gaps). Now tunnelling may take place through the tunnelling bonds in various ways, so that the functional form of the tunnelling current as a nonlinear function of the potential difference across them may be quite complicated. For simplicity, we address the aspects of nonlinearity in a macroscopic system which comes through two piecewise linear regions of a tunnelling element. The piecewise linear transport is in fact a highly nonlinear process as there is a cusp singularity at the intersection point. The transport due to tunnelling which is the source of nonlinearity in the experimental systems [13, 14] we focus on, can be well approximated in this way and thus the nonlinearity of the macroscopic systems may be understood at a qualitative (and, sometimes even at a quantitative) level. Next, one notes as discussed above that in many physical systems, the response is negligibly low (or there is no response at all) until and unless the driving force exceeds a certain threshold value. So a class of problems exist where sharp thresholds to transport occur. The examples in the electrical case are a Zener diode, a CDW system or a typeII superconductor and in the fluid permeability problems, for example, is a Bingham fluid (where there is a critical shear stress $\tau_{c}$, above which it has a finite viscosity and below which it is so enormously viscous that it does not flow). In our RRTN model, we work with tunnelling bonds which have zero conductance below a threshold.

But, our percolative model is not just a random mixture of two phases. For our convenience we take a square lattice in $2 \mathrm{D}$. The basic physics should remain the same if we go over to 3D. Conducting/ ohmic bonds (o-bonds) are thrown at random at a certain volume fraction $p$. The rest $(1-p)$ fraction contains insulators. Now we allow tunnelling bonds ( $t$-bonds) only across the nearest-neighbour $(n n)$ gaps of two conducting bonds (and no further) if an appropriate voltage is applied externally across two opposite sides of such a random resistor cum tunnelling network (RRTN). Our interest is to examine this proposed correlated percolation model in the spirit of dielectric breakdown phenomenon. The mechanism operating here is clearly not 
traditional dielectric breakdown because the piecewise linear response considered here in the tunnelling bonds is reversible in the sense that if the local voltage difference is lowered below the threshold a tunnelling bond becomes insulating again. This is an important point because if we would assume the process to be irreversible, then the irreversible conversion of one insulating element to a conducting one may trigger an avalanche effect. Since a local current redistribution takes place in the reversible RRTN model as well whenever a dielectric turns metallic, avalanche may take place in this model as well, but the avalanches may be more restricted in the RRTN than the traditional reversible models. Similarly in the random fuse network [11] one has the irreversibility with respect to conductor $\rightarrow$ insulator transtion with the increase of applied field. Breaking (fusing) of one bond in a certain path permanently (because of too much stress) may lead to an increase of current density in the other paths and thus it may trigger an avalanche effect. Since a local current re-distribution takes place in the reversible RRTN model as well whenever a dielectric turns metallic, avalanche may take place in this model too, but the avalanches may be more restricted in the RRTN than in the traditional 'irreversible' models. In practice the 'reversibility' situation is achieved when the charge transport by tunnelling gives the most important contribution to the breakdown process than the permanent breakdown of the microscopic conductors/ insulators inside the system. One example of this is the experiment on dielectric breakdown demonstrated by Benguigui et al. [12 where the authors consider a network of tunnelling diodes. There are many other real systems demonstrating this reversibility, e.g., carbon-wax mixture [13], $\mathrm{Ag}$ - $\mathrm{KCl}$ composite [14] and many other nonlinear composites where the macroscopic $I$ - $V$ characteristic is reversible (no appreciable hysteresis effect).

Next we comment on the procedure for obtaining the breakdown voltage $\left(V_{B}\right)$ for the usual dielectric breakdown problem as understood from the references above. The usual procedure to obtain the electrostatic voltage distribution at the nodes of the networks in the non-percolating situation is to solve for the Laplace's equation 
$\left(\nabla^{2} V=0\right)$. This procedure, when discretised on a square lattice and in the situation where the dielectric constant for all the bonds are assumed to be the same (pure dielectric), reduces to $v_{0}=\sum v_{i} / 4$, where $v_{0}$ is the voltage at any node and $v_{i}$ 's are the voltages at the four nearest-neighbour $(n n)$ nodes on a square lattice. In our case, we approach the breakdown point from the conducting side and apply Kirchhoff's law for our problem which takes the form: $v_{0}=\sum v_{i} g_{i} / \sum g_{i}$, where $g_{i}$ 's are the conductances of the $n n$ bonds. Clearly this may be reduced to the discrete Laplace's equation above had the $g_{i}$ 's for all the bonds been essentially the same. Further, in the usual models, as soon as the voltage difference $\left(v_{i}-v_{0}\right)$ across an insulating bond exceeds its threshold value $v_{g}$, this bond is turned into a 'perfect' conductor for all later time (iterations) to come, and $v_{i}$ is made equal to $v_{0}$. On the other hand, in our model, even when a t-bond has been broken (turned metallic), neither does it become a perfect conductor nor does it carry any carry any current until and unless the voltage difference across it exceeds $v_{g}$. We believe that this is a crucial difference and should be more akin to reality.

As per our model is concerned we assume that the tunnelling bonds (the bonds which break) may be placed only in the $n n$ gaps of two conducting bonds and nowhere else. It will be noted that because of the reversible nature of our $t$-bonds and their finite thresholds $v_{g}$, rarely would $V_{I}$ be equal to $V_{F}$ in our model. Indeed we $d o$ not work with $V_{I}$ and actually identify $V_{B}$ as the average of the final breakdown voltages $V_{F}$. Hence a typical breakdown path in the RRTN model consists of an actual number of the so-called 'broken' bonds and does not quite correspond to the minimum gap path except when $p$ is very close to $p_{c}$. If there are $n$ number of active tunnelling (or broken) bonds in the minimum gap path having a threshold voltage $v_{g}$ for each of them, the overall breakdown voltage $V_{B}=n v_{g}$. It may be noted that this is also the case with the dielectric breakdown experiment by Benguigui et al. [12] on an artificially constructed electrical network of resistors and light emitting diodes (LED). The initial breakdown voltage $V_{I}$ (at which at least one tunnelling bond breaks) is 
just $v_{g}$. Very rarely (except for $p$ near $p_{c}$ ) one has $n=1$, and $V_{B}=V_{I}$ in our model or in the above mentioned experiment by Benguigui et al. As a demonstration, we show here a typical configuration (see fig. 1) of the lattice of size $L=10$ at a volume fraction $p=0.30$ where just one breakdown path has been formed. Indicated by the dashed lines are the number of broken $t$-bonds. The path is explicitly seen to not be the minimum gap path.

One may notice another difference of our model from the usual models of dielectric breakdown problems so far studied (where the dielectric bonds can break at any place in the network) from the above demonstration. There may be a series of broken bonds at more than $n n$ gaps of two conducting bonds in the breakdown path in the usual model (see, e.g., the figures in ref. [6]) but not in ours. It is worth commenting here that the breakdown paths generated by Benguigui et al. [12] are more akin to our model than the usual model. This is because even though many more than one LED's seem to be broken in series, in practice two consecutive LED's are connected by metallic wires and hence do not correspond to breakdown over two or more near neighbour distances (or lattice constants). The breakdown exponent $\left(t_{B}\right)$ in this experiment was reported to be $\cong 1.1$, which is smaller than what is actually expected (i.e., 4/3, the exact value of $\nu$ in 2D). The difference may be attributed to the finite size effect since a system of size $20 \times 20$ was considered.

\section{Calculation of the Breakdown exponent in the RRTN}

Here we examine the dielectric breakdown phenomenon in our model as the onset of nonlinear conduction against applied field for $p \leq p_{c}$. Below the percolation threshold $\left(p_{c}\right)$ there exists a number of metallic clusters, isolated from each other, but closely spaced. The conductivity is a sensitive function of applied electric field/ voltage 9 9 as new conducting paths are created when the applied external electric field increases 
above the dielectric breakdown field $\left(E_{B}=V_{B} / L\right)$ of the insulator. Note that the model has a percolation threshold at $p_{c t} \cong 0.18$ [15] if all the tunnelling bonds overcome their voltage thresholds at the appropriate positions. So below $p_{c t}$ there is no sample-spanning cluster of combined $o$ - and $t$-bonds, and hence there is no conduction (on an average) at any finite electric field according to the criterion set for our model. Thus three types of configurations arise in the regime $p_{c t}<p<p_{c}$ :

- Some configurations which are already percolating with the ohmic bonds only: they have zero voltage threshold macroscopically,

- Some configurations which donot percolate with the ohmic bonds only but do so in conjunction with the tunnelling bonds: they have a finite voltage threshold,

- Some other configurations are there which never percolate even with the assistance of all the available tunnelling bonds: they do not take part in the breakdown process.

This third possibility does not arise in the usual class of breakdown problems where any insulating bond may break given enough voltage and hence eventually renders the system conducting.

Clearly, to find the average breakdown voltage $\left(V_{B}\right)$ we have to disregard those configurations which do not take part in the breakdown phenomenon. In the fig. 2, a typical distribution of breakdown voltages $V_{B}$ is shown for a system size $L=40$ and $p=0.45$. This distribution is quite broad and seems to be asymmetric.

The phase diagram is shown in the fig. $\mathbf{3}$ as the average of breakdown voltage $\left(V_{B}\right)$ plotted against the volume fraction $(p)$ of conducting bonds. This typical figure is shown for a system of size $L=30$ and average is taken over 500 configurations. Our interest would be to know how does the average breakdown field $\left(E_{B}=V_{B} / L\right)$ scale against $\left(p_{c}-p\right)$ as in eqn. (11). One usually plots the quantity $V_{B}$ or $E_{B}$ for a finite sized system against $\left(p_{c}-p\right)$ around $p_{c}$ in log-log scale and find out the breakdown exponent $t_{B}(L)$ from the least square fit. To remove the finite-size effects, however, 
we follow a slightly different way of finding the above exponent. We first obtain the finite size scaling of the breakdown field, $E_{B}$. One such scaling plot is shown in fig. 4 for $p=0.4$. In this way, we obtain the asymptotic values $E_{B}(L=\infty)$ of the breakdown field for all $p$ ranging from 0.3 to 0.5 through finite size scaling, which seems to follow

$$
E_{B}^{p}(L)=E_{B}^{p}(\infty)+a(p) L^{-\mu(p)},
$$

where $\mu\left(p_{c}\right) \simeq 1$; but quite different (0.4 to 0.75$)$ at other $p<p_{c}$. Further $E_{B}^{p_{c}}(\infty)$ has a very small but positive value which for the accuracy of our calculation implies that $E_{B}^{p_{c}}(\infty)=0$. But as $p$ becomes smaller and smaller than $p_{c}, E_{B}^{p}(\infty)$ increases systematically as the graph in fig. 5 indicates. We point out that forcing $E_{B}^{p}(\infty)=$ 0 at $p<p_{c}$ gives significantly worse fitting. Eqn. (2) strongly demonstrates the fact that the breakdown model we are considering is somewhat different in nature from the usual models available in the literature where one observes a $1 / \ln L$ scaling of $E_{B}^{p}$ demonstrated clearly in the work of Beale and Duxbury [6]. This scaling, which makes the $E_{B}^{p}$ 's vanish irrespective of the $p$ in a truly infinite size system, is nonexistent in our model. Since the breakdown field in the previous models vanishes to zero irrespective of any $p\left(p<p_{c}\right)$, it is worth noting that the above $1 / \ln L$ scaling and the consequent vanishing of $E_{B}^{p}$ is also non-existent in another model which has no dilution but has reversible tunneling conductors with random thresholds at each and every bond in the lattice. In such a network, Roux and Herrmann [16] found that $V_{B}=(0.22 \pm 0.02) L$.

The scaling of the asymptotic breakdown field $E_{B}^{p}(\infty)$ can be written as

$$
E_{B}^{p}(\infty) \sim\left(p_{c}-p\right)^{t_{B}}
$$

The double logarithmic plot of eqn. (3) is shown in fig. 6 and the least square fit of the data is also shown. We find from this fitting that the breakdown exponent $t_{B} \cong 1.42$ for our RRTN model. 


\section{Discussion}

It seems that the above exponent $t_{B}$ is not very different from that of the usual breakdown exponent $t_{B}=\nu=1.33$ as discussed above. But it is not unlikely either that we do indeed have a different result in our hands. If different, it could be because of the nature of the electric field in increasing the effective volume fraction of the conductors. As may be understood, the electric field adds on new bridge bonds (active $t$-bonds) at well-determined positions (according to the deterministic laws of electricity) which must be completely different from the random positions of the extra $o$-bonds obtained by increasing the volume fraction to the same effective volume fraction as obtained by applying the electric field. Intuitively, the correlations obtained by these two different means should be qualitatively different (one being isotropic and the other anisotropic). Indeed, as seen in an experiment 12 as well as in simulations [6, 17] (see also fig. 1), an electric field tends to make somewhat elongated clusters directed towards the direction of the external field. But, our results in ref. [17] do also show that while the directivity (anisotropy) of the clusters increases with an increasing field upto a maximum, it does finally start to decay (i.e., grows more and more isotropic) at still larger fields and the RRTN at an infinite field, which becomes our fully correlated bond percolation model [15], does not fall in the category of directed percolation (rather it falls in the same universality class as the ordinary random bond percolation). Thus, at a small but finite field, we may observe the percolation statistics to be directed only a little bit. Now, it is well-known from the results on directed percolation that the correlation length exponent in a direction parallel to the electric field is $\nu_{\|} \cong 1.7$. So, it is not unlikely that the correlation length exponent near the breakdown field (which is quite small) takes some value between 1.3 and 1.7. If true, this may very well explain why out $t_{B}=1.42$. In this respect, it may be noted that Beale and Duxbury [6] also found the average $t_{B}=1.46$. Thus, the exponent $t_{B}=1.42$ for our model may actually be a result different from 
the standard quoted result of $4 / 3$ for this exponent. As a final remark we would like to add that it would be worthwhile to take the usual model for breakdown and repeat the calculation for the final breakdown exponent under the condition that the dielectrics are reversible (i.e., that they are not broken permanently) and compare the breakdown exponent with the one obtained here.

\section{References}

[1] see the articles in Statistical Models for the Fracture of Disordered Media, eds. H.J. Herrmann and S. Roux, (North-Holland, 1990)

[2] D. Stauffer, Physica A, 1997: to appear

[3] D.R. Bowman and D. Stroud, Bull. Am. Phys. Soc. 30, 563 (1985); ibid Phys. Rev. B 40, 4641 (1989); P.M. Duxbury, P.D. Beale and P.L. Leath, Phys. Rev. Lett. 57, 1052 (1986)

[4] P. Ray and B.K. Chakrabarti, J. Phys. C 18, L185 (1985); Solid State Commn. 53, $477(1985)$

[5] S.S. Manna and B.K. Chakrabarti, Phys. Rev. B 36, 4078 (1987)

[6] P.D. Beale, P.M. Duxbury, Phys. Rev. B, 37, 2785 (1988)

[7] R.B. Stinchcombe, P.M. Duxbury and P. Shukla, J. Phys. A 19, 3903 (1986); B.K. Chakrabarti, K.K. Bardhan and P. Ray, J. Phys. C 20, L57 (1987)

[8] J.T. Chayes, L. Chayes and R. Durret, J. Stat Phys. 45, 933 (1986)

[9] A.K. Sen and A. Kar Gupta in Non-linearity and Breakdown in Soft Condensed Matter, eds. K.K. Bardhan, B.K. Chakrabarti and A. Hansen, Lecture Notes in Physics, 437, 271 (Springer-Verlag, Berlin 1994) 
[10] Y. Gefen, W-H. Shih, R.B. Laibowitz and J.M. Viggiano, Phys. Rev. Lett. 57, 3097 (1986)

[11] L. de Arcangelis, S. Redner and H.J. Herrmann, J. Physique Lett. 46, L-585 (1985)

[12] L. Benguigui and P. Ron in Non-linearity and Breakdown in Soft Condensed Matter, eds. K.K. Bardhan, B.K. Chakrabarti and Alex Hansen, Lecture Notes in Physics, 437, 271 (Springer-Verlag, Berlin 1994)

[13] R.K. Chakrabarty, K.K. Bardhan and A. Basu, J. Phys.: Condens. Matt. 5, 2377 (1993); K.K. Bardhan and R.K. Chakrabarty, Phys. Rev. Lett. 69, 2559 (1992)

[14] I-G. Chen and W.B. Johnson, J. Mat. Sc. 27, 5497 (1992)

[15] A. Kar Gupta \& A.K. Sen, Physica A 215, 1 (1995)

[16] S. Roux and H.J. Herrmann, Europhys. Lett. 4, 1227 (1987)

[17] A. Kar Gupta \& A.K. Sen, J. Stat. Phys. 80, 1425 (1995) 


\section{Figure Captions}

Fig.1 A typical configuration of the lattice for a square of size $10 \times 10$ with $p=$ 0.3 (below $p_{c}$ ). The breakdown path is indicated by 'abcd' with $n=4$ which is seen to be different from the minimum-gap path 'aef' of an usual dielectric breakdown model with $g(p)=3$.

Fig.2 A typical distribution of breakdown voltage $V_{B}$ with $v_{g}=0.5$.

Fig.3 The phase diagram for the dielectric breakdown is shown with the average of breakdown voltage $\left(V_{B}\right)$ plotted against the volume fraction $(p)$ of conducting bonds for a square lattice with $L=30$.

Fig.4 The finite size scaling of breakdown field $E_{B}$.

Fig.5 The behaviour of asymptotic breakdown field $E_{B}^{p}(\infty)$ with $p$.

Fig.6 The $\log -\log$ plot of $E_{B}^{p}(\infty)$ against $\left(p_{c}-p\right)$ and the best fit line to find the breakdown exponent. The fitted line gives $t_{B}=1.42$. 


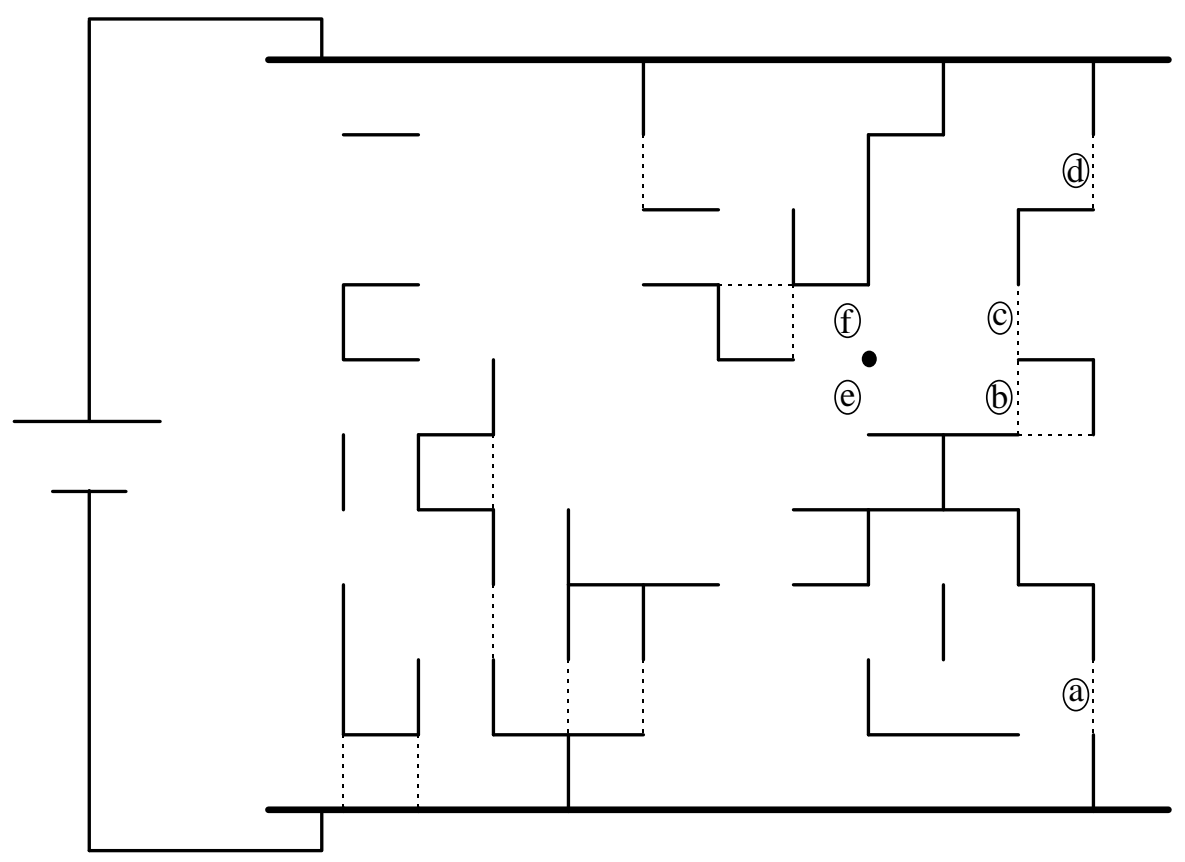




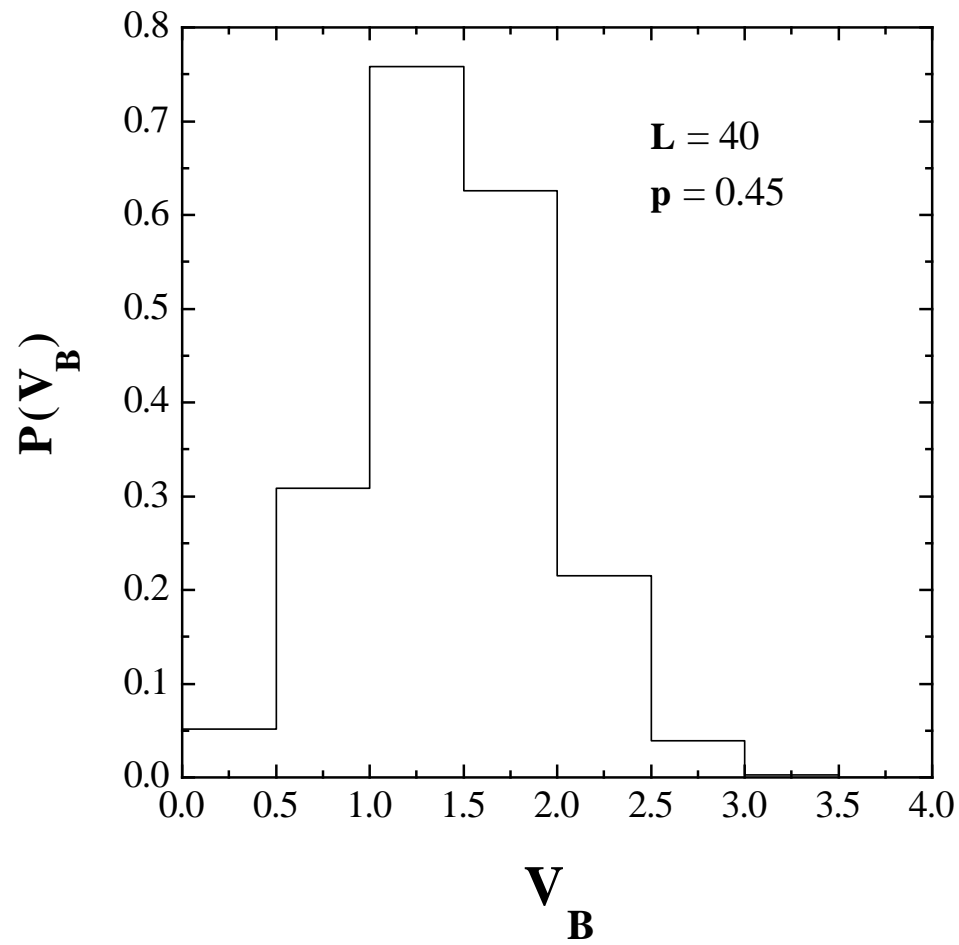




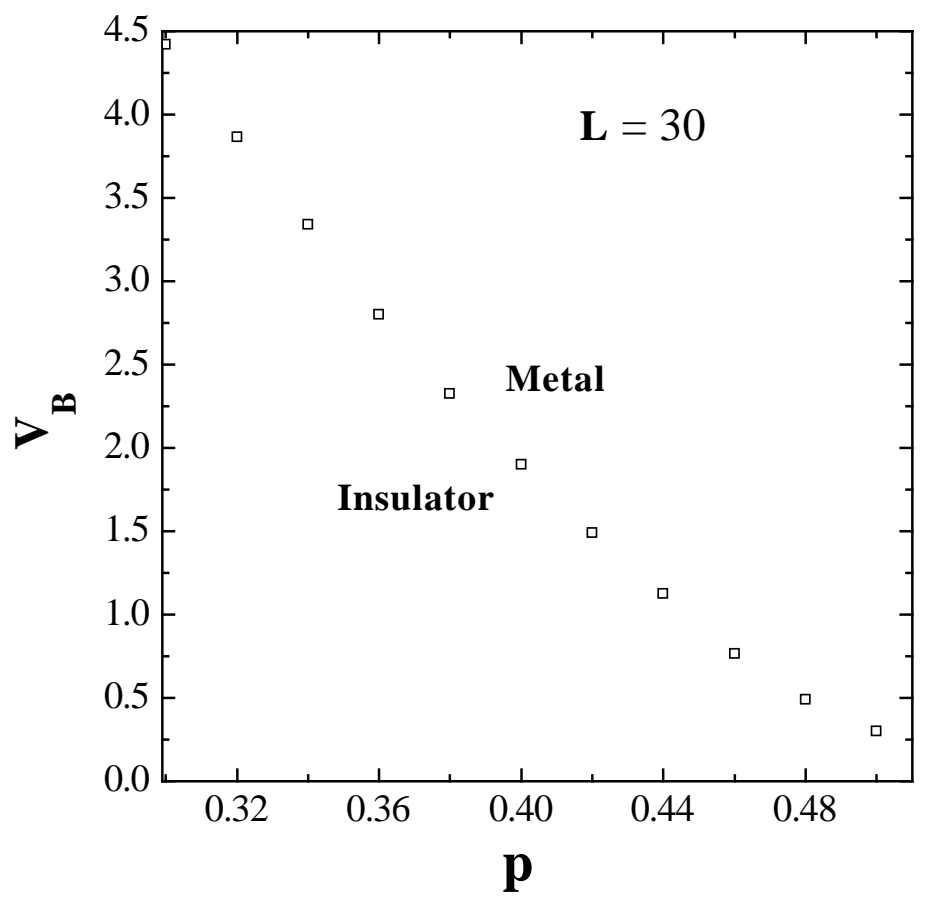




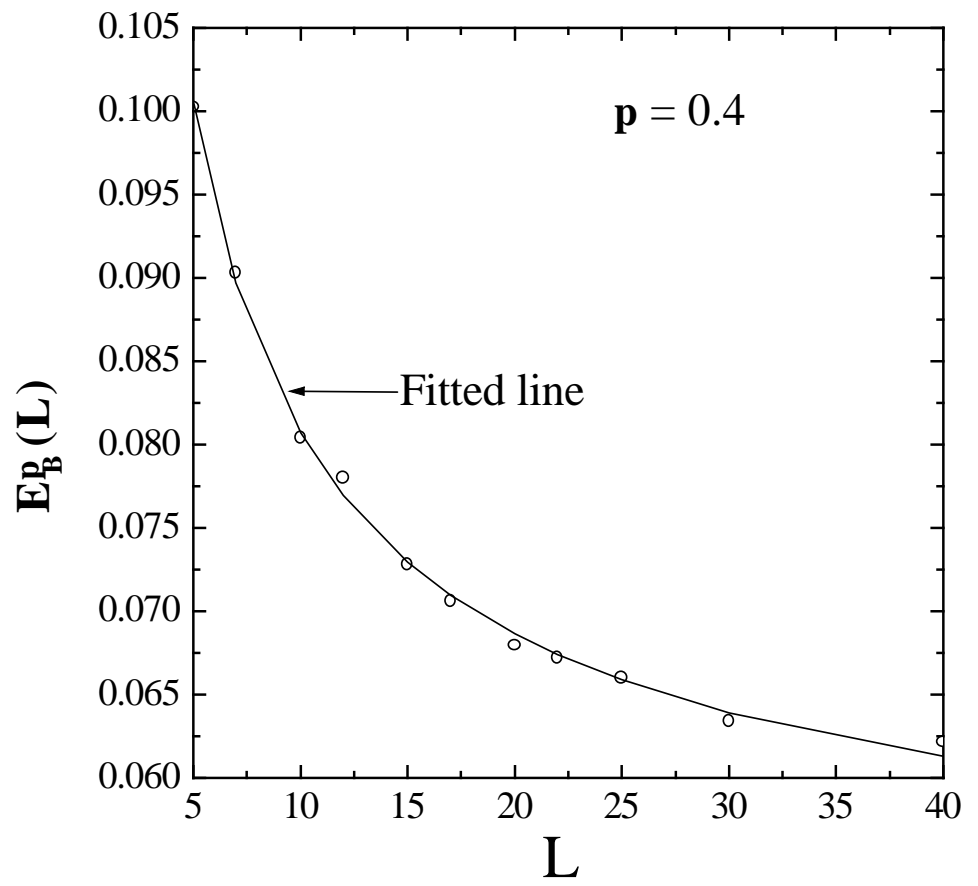




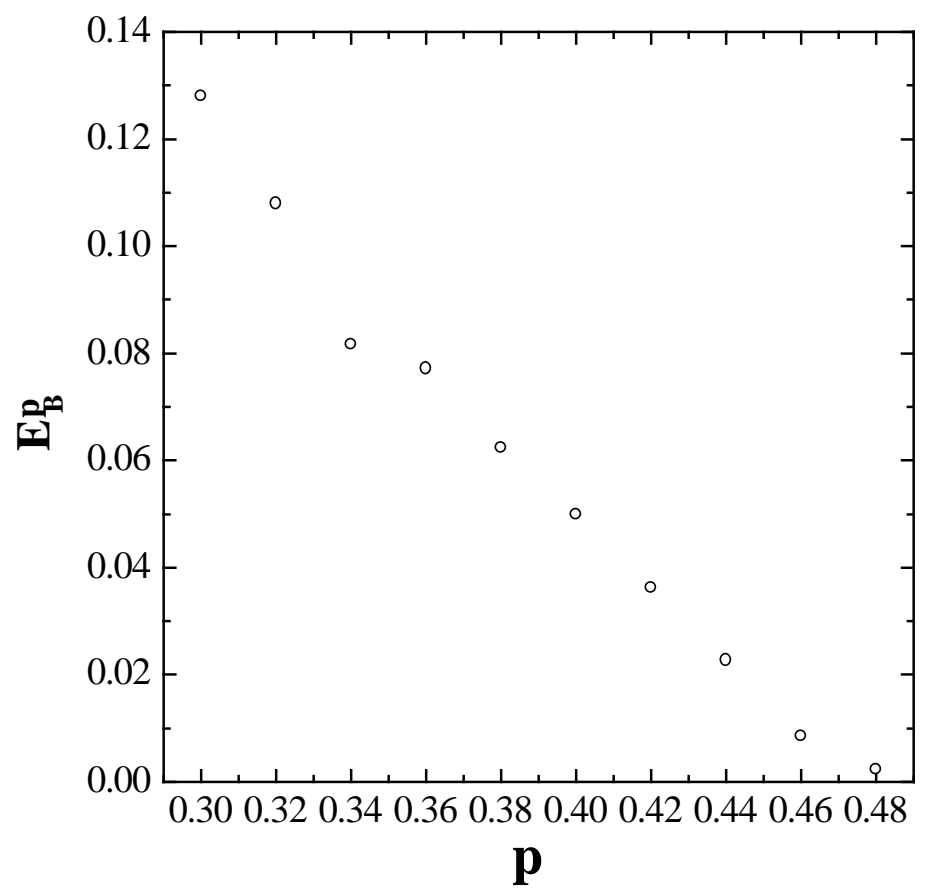




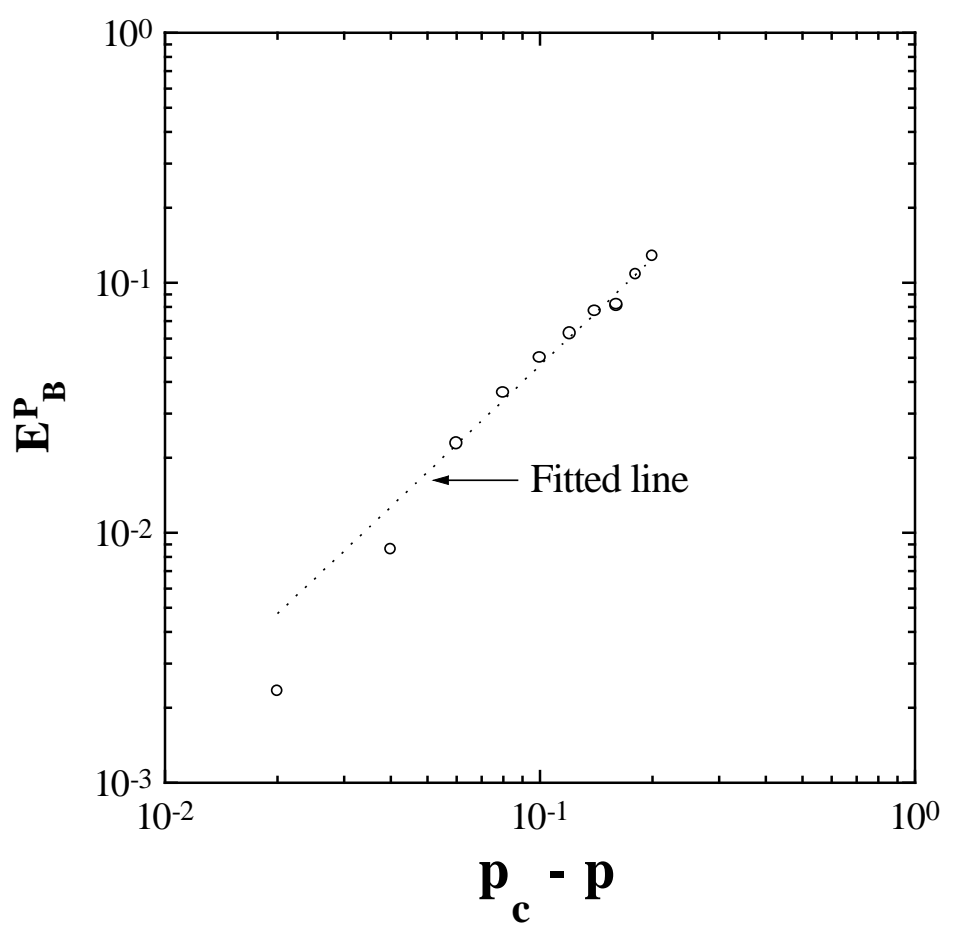

